\title{
The inner environment of $Z$ Canis Majoris: High-contrast imaging polarimetry with $\mathrm{NaCo}^{\star}$
}

\author{
H. Canovas ${ }^{1,6}$, S. Perez ${ }^{2,6}$, C. Dougados ${ }^{3}$, J. de Boer ${ }^{4,7}$, F. Ménard ${ }^{3}$, S. Casassus ${ }^{2,6}$, M. R. Schreiber ${ }^{1,6}$, \\ L. A. Cieza ${ }^{5,6}$, C. Caceres ${ }^{1,6}$, and J. H. Girard ${ }^{7}$ \\ 1 Departamento de Física y Astronomía, Universidad de Valparaíso, Blanco 951, Valparaíso, Chile \\ e-mail: hector.canovas@dfa.uv.cl \\ 2 Departamento de Astronomía, Universidad de Chile, Casilla 36-D, Santiago, Chile \\ 3 UMI-FCA, CNRS/INSU, France (UMI 3386), and Dept. de Astronomía, Universidad de Chile, Santiago, Chile \\ 4 Sterrewacht Leiden, Universiteit Leiden, PO Box 9513, 2300 RA Leiden, The Netherlands \\ 5 Facultad de Ingeniería, Universidad Diego Portales, Av. Ejercito 441, Santiago, Chile \\ 6 Millennium Nucleus "Protoplanetary Disks in ALMA Early Science", Universidad de Valparaiso, Valparaiso, Chile \\ 7 European Southern Observatory, Casilla 19001, Santiago, Chile
}

Received 18 March 2015 / Accepted 20 April 2015

\section{ABSTRACT}

\begin{abstract}
Context. ZCMa is a binary composed of an embedded Herbig Be and an FU Ori class star separated by $\sim 100$ au. Observational evidence indicates a complex environment in which each star has a circumstellar disk and drives a jet, and the whole system is embedded in a large dusty envelope.

Aims. We aim to probe the circumbinary environment of ZCMa in the inner 400 au in scattered light.

Methods. We use high-contrast imaging polarimetry with VLT/NaCo at the $H$ and $K_{\mathrm{s}}$ bands.

Results. The central binary is resolved in both bands. The polarized images show three bright and complex structures: a common dust envelope, a sharp extended feature previously reported in direct light, and an intriguing bright clump located 0!' 3 south of the binary, which appears spatially connected to the sharp extended feature.

Conclusions. We detect orbital motion when compared to previous observations, and report a new outburst driven by the Herbig star. Our observations reveal the complex inner environment of ZCMa in unprecedented detail and contrast.
\end{abstract}

Key words. stars: variables: T Tauri, Herbig Ae/Be - binaries: general - stars: pre-main sequence - accretion, accretion disks instrumentation: high angular resolution - techniques: polarimetric

\section{Introduction}

Herbig stars are the earliest optically revealed stage of mediummass star formation. They are usually found embedded in envelopes rich in gas and dust, typically surrounded by circumstellar protoplanetary disks. Stellar multiplicity is an important element that has a strong impact on circumstellar evolution. Interestingly, most stars are born in multiple systems (Kraus et al. 2012). Z Canis Majoris (ZCMa) is an enigmatic binary system composed of an embedded Herbig Be star (primary) and an FU Ori (secondary) star separated by 100 au (Koresko et al. 1991). Distance estimations to ZCMa range from 930 to 1150 pc (Clariá 1974; Kaltcheva \& Hilditch 2000). The whole system is surrounded by a huge infalling envelope (Beckwith \& Sargent 1991; Alonso-Albi et al. 2009), which can explain the very high accretion rate of the FU Ori star $\left(\sim 3 \times 10^{-5} M_{\odot} \mathrm{yr}^{-1}\right.$, van den Ancker et al. 2004). Spectropolarimetric observations of ZCMa revealed the nature of the primary showing a spectrum rich in emission lines and largely polarized (Whitney et al. 1993). A dusty envelope or cocoon around the primary is most likely causing this effect. The extinction $\left(A_{v} \sim 10(5.2)\right.$ for the primary (secondary), Hinkley et al. 2013) created by this cocoon can decrease the brightness of the primary towards optical

\footnotetext{
* Based on observations made with the VLT, program 094.C-0416(A).
}

wavelengths (see also Whitney \& Hartmann 1993; Szeifert et al. 2010). The primary drives a 3.6 pc jet (Poetzel et al. 1989), and IFU observations with OSIRIS/Keck show that the secondary drives a "microjet" ( 400 au, Whelan et al. 2010). At a few hundred au south of Z CMa, Millan-Gabet \& Monnier (2002) reported the presence of a jet-like feature observed in scattered light extending about $\sim 1000$ au from Z CMa towards the southwest. It is not clear whether it is associated with a bipolar outflow, or a jet, or whether it is a completely independent structure.

The ZCMa light curve shows strong and moderate outbursts with variations both in duration and in strength. During quiescence the secondary dominates the optical and near-infrared (IR) up to the $H$ band and the primary dominates at longer wavelengths. However, during the strong outburst episodes the Herbig star becomes the dominant source at all wavelengths (van den Ancker et al. 2004; Hinkley et al. 2013). Between 2008 and $2009 \mathrm{ZCMa}$ experienced a strong outburst $\left(\Delta m_{v} \approx 2.5\right)$. While some observational evidence favors an accretion burst from the primary as the source of this outburst (Benisty et al. 2010; Hinkley et al. 2013), other observations favor the formation of holes in the dust cocoon around the primary to explain the sudden increment in flux (Szeifert et al. 2010). Changes in the structure and/or optical thickness of the dust cocoon can naturally explain the outburst: the opening of new holes in the direction of the line of sight allows the light from the Herbig star 
to directly escape, resulting in a dramatic increment of the total flux of the system at optical and NIR wavelengths. Canovas et al. (2012) presented polarized differential images (PDI) at optical wavelengths, showing evidence of light escaping through a hole in the dust cocoon and cavities carved out by the two jets in the common dusty envelope surrounding the system.

In this Letter we present high-contrast PDI observations of $\mathrm{ZCMa}$ at the $H$ and $K_{\mathrm{s}}$ bands with $\mathrm{NaCo}$. Our results show the excellent high-contrast capabilities of this instrument after its implementation from VLT/UT4 to VLT/UT1. We find new polarized features down to an inner working angle of $\sim 0$ !' 12 , and resolve the two components of ZCMa in the two bands showing that the primary is driving a new outburst. Our results allow us to describe the complex inner environment of this young system in unprecedented detail.

\section{Observations}

We observed ZCMa and the comparison star HD 52841 with $\mathrm{NaCo}$ (Lenzen et al. 2003; Rousset et al. 2003) on 19 January 2015, right after its commissioning at the VLT/UT1. The seeing was mostly stable (median $\sim 0$ ' 87 ) during the observations with a few short episodes of bad values (above 1". 10) and a median coherence time of $\tau_{0}=3.5 \mathrm{~ms}$. ZCMa was very bright $\left(m_{H} \leq 6\right)$ and the adaptive optics (AO) system delivered near diffraction limited images.

Our observations were obtained with the NIR detector with the $H$ and $K_{\mathrm{s}}$ filters. The S27 camera $\left(27.05 \pm 0.1 \mathrm{mas} \mathrm{px}^{-1}\right)$ was used in cube mode with the read-out set to Double_RdRstRd and the detector mode set to HighDynamic. We used individual detector integration times (DIT's) of $0.15 \mathrm{~s}$ and $0.5 \mathrm{~s}$ for ZCMa, and $1 \mathrm{~s}$ for HD 52841. The frame loss rate in the short exposure images was minimized by windowing the read-out region to $512 \times 520 \mathrm{px}$. The effect of bad pixels was reduced by dithering on the detector. HD 52841 was below the non-linear $\mathrm{NaCo}$ regime $\left(10^{4}\right.$ counts) for the entire observation time. For $\mathrm{ZCMa}$, the primary is saturated in all our images and several pixels surrounding both stars have values above the linear regime. We used NaCo in polarimetry mode combining a half-wave plate (HWP) with a Wollaston prism. In this setup the HWP rotates the polarization plane of the incoming light and the Wollaston splits the light beam into two images with orthogonal polarization states that are projected in different regions of the detector. A field mask avoids beam-overlapping. These two images are hereafter called $I_{\mathrm{o}}$ (for the ordinary beam) and $I_{\mathrm{e}}$ (for the extra-ordinary beam). We took images with the HWP rotated by $0^{\circ},-22.5^{\circ},-45^{\circ}$, and $-67.5^{\circ}$ to reconstruct the Stokes parameters of the incoming light. The observations are listed in Table 1.

\section{Data reduction}

We used our own pipeline outlined in Canovas et al. (2011, 2013) to process the observations. Here we briefly describe the major aspects of the reduction. The images with the poorest $\mathrm{AO}$ correction (obtained under seeing $>11^{\prime \prime} 1$ ) were discarded in our analysis. The rest of the images were dark subtracted, flat-field corrected, and sky-background subtracted. Hot and dead pixels were flagged. The images were first aligned with a crosscorrelation algorithm. We then refined the alignment up to an accuracy of $0.05 \mathrm{px}$ with a minimization method. In short, our algorithm finds the position that minimizes the standard deviation $(\sigma)$ of the difference between the two images to be aligned using cubic interpolation. The polarized information was extracted using the double-difference approach (e.g. Hinkley et al. 2009; Canovas et al. 2011). This way the $I_{\mathrm{o}}$ and $I_{\mathrm{e}}$ images are
Table 1. Observing log of the observations.

\begin{tabular}{ccccc}
\hline Target & Band & $\begin{array}{c}\text { DIT } \\
{[\mathrm{s}]}\end{array}$ & $\begin{array}{c}\text { Tot. exp. time } \\
{[\mathrm{s}]}\end{array}$ & $\begin{array}{c}\text { Airmass } \\
{[\mathrm{min}, \mathrm{max}]}\end{array}$ \\
\hline $\mathrm{ZCMa}$ & $H$ & 0.15 & 957.9 & $1.03,1.15$ \\
& & 0.5 & 166.0 & $1.04,1.05$ \\
& $K_{\mathrm{s}}$ & 0.15 & 603.0 & $1.05,1.07$ \\
$\mathrm{HD} 52841$ & & 0.5 & 176.0 & $1.07,1.10$ \\
& $H$ & 1 & 160.0 & $1.20,1.22$ \\
& $K_{\mathrm{s}}$ & 1 & 160.0 & $1.24,1.26$ \\
\hline
\end{tabular}

Notes. Bad seeing frames do not contribute to the total exposure time listed below. All observations were taken on 19 January 2015.

added and subtracted to produce an intensity and polarized image, respectively. When the HWP is at $0^{\circ}$ the polarized image corresponds to the Stokes $+Q$ image. Similarly, when the HWP is at $22.5^{\circ}, 45^{\circ}$, and $67.5^{\circ}$ the resulting polarized image corresponds to Stokes $+U,-Q$, and $-U$, respectively. Instrumental polarization (IP) is usually corrected at this stage assuming that the central star is unpolarized (e.g., Avenhaus et al. 2014). Unfortunately, this is not the case for the two stars in the Z CMa system (Fischer et al. 1998). Additionally, the saturation of the primary and the extended detector area around the two stars with pixel values well above the non-linear regime of the $\mathrm{NaCo}$ detector complicate the polarimetric data reduction. We therefore measure the polarization degree in a 1-px thick $\left(0.0^{\prime} 027\right)$ contour around the non-linear region for each individual image. This region contains contributions from the IP and the interstellar polarization and reaches a maximum of $\sim 2.5 \%{ }^{1}$ in our observations. This quantity was subtracted from the individual images. To test for the effect of this correction we also reduced our images using different amounts of correction ranging from $1 \%$ to $4 \%$. The non-linear pixels are flagged and not considered in our polarimetric analysis. The images were median-combined to construct the final Stokes parameters as $Q=(+Q-(-Q)) / 2$ and $U=(+U-(-U)) / 2$. The (linearly) polarized intensity $\left(P_{I}\right)$ is described by $P_{I}=\sqrt{Q^{2}+U^{2}}$. The polarization angle indicating the vibration plane of the electric field is $P_{\theta}=\frac{1}{2} \arctan (U / Q)$. The total intensity is computed by adding up the median-combined intensity images. Finally, the images were normalized to $1 \mathrm{~s}$ exposure time.

\section{Results}

The short-exposed images of ZCMa show the same structures as the long-exposure ones while having smaller inner working angles. Therefore, we focus our analysis on the $0.15 \mathrm{~s}$ images. In what follows we adopt the most recent value of $930 \mathrm{pc}$ for the distance to the source.

The ZCMa binary is resolved in both $H$ and $K_{\mathrm{s}}$ bands (Fig. 1 upper panels). The primary component is saturated in both bands. To estimate the separation $(\rho)$, position angle (PA), and difference in flux $\left(\Delta m_{H, K \mathrm{~s}}\right)$ of the ZCMa binary system is not straightforward because of the saturation of the primary and the small separation between both stars. We circumvent this problem by fitting the primary's point spread function (PSF) wings and the secondary's full PSF. This way we derive a separation of $\rho=0 \prime^{\prime} 112 \pm 0 .^{\prime} 003$ and a PA of $139.6^{\circ} \pm 2.0^{\circ}$. The brightness difference between the primary and secondary is noticeably larger at the $K_{\mathrm{s}}$ than at the $H$ band, with $\Delta m_{H}=-1.1 \pm 0.2$ and

Witzel et al. (2011) reports a maximum IP for $\mathrm{NaCo}$ at UT4 of $4 \%$. 


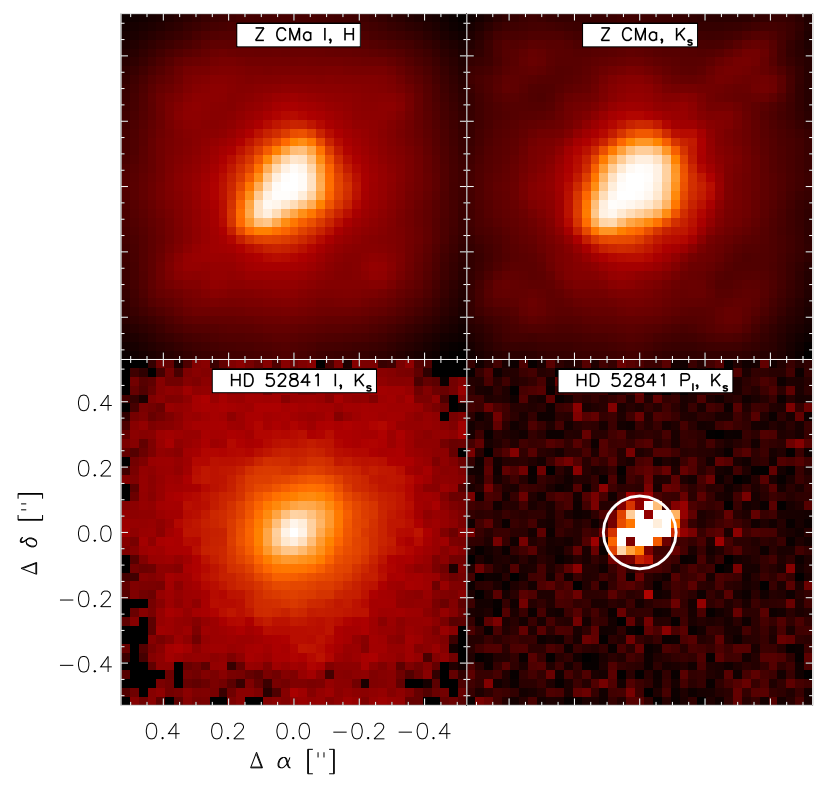

Fig. 1. Top-left and bottom-left: intensity images in logarithmic scale. $\mathrm{AO}$ artifacts are visible in the Z CMa images. HD 52841 is slightly elongated in the SE-NW direction. Bottom-right: the $P_{I}$ image of HD 52841 shows the noise that inevitably appears in the innermost $0 . ' 1$ of the image (indicated by the white circle) as a result of imperfect speckle suppression.

$\Delta m_{K_{\mathrm{s}}} \widetilde{<}-1.9$, similar to the values observed during an outburst driven by the primary (Hinkley et al. 2009; Szeifert et al. 2010).

Comparing our results with those derived by Millan-Gabet \& Monnier (2002) using data from 2001 we detect an orbital motion of $\Delta \mathrm{PA}=10.6^{\circ} \pm 2.1^{\circ}$ and $\Delta \rho=0 .^{\prime} 003 \pm 0{ }^{\prime} 003$ in $14 \mathrm{yr}$. Our results are in agreement with Millan-Gabet \& Monnier (2002) who find a marginal change in $\rho$ but a significant change of $\Delta \mathrm{PA}=8.8 \pm 1.5$ when analyzing previous observations covering a $11.2 \mathrm{yr}$ baseline, deriving a $\triangle \mathrm{PA}$ rate of $\sim 0.7 \mathrm{yr}^{-1}$ for a circular orbit composed of a $1 M_{\odot}$ and a $5 M_{\odot}$ star.

Our polarized images (Fig. 2) show three complex features down to the inner $\sim 0$.' 12 from the stars in the two bands. First, we detect an approximately circular halo around the two stars (labeled as "1" in Fig. 2, left panel). The detector window trims the upper part of the image, where the halo is detected at a signalto-noise ratio $(\mathrm{S} / \mathrm{N})$ of $\sim 8$, suggesting that it extends beyond that limit. In the east-west direction the halo is detected up to $\sim 0$ ' $^{\prime} 8$ ( $744 \mathrm{au}$ ) from ZCMa. Second, we detect a bright, irregularly shaped polarized clump at $\sim 0 ! 3$ ( $\sim 279$ au projected distance) south from ZCMa (labeled as " 2 "). This clump is elongated in the east-west direction and it is separated by roughly 0.15 from the "k1" feature described by Whelan et al. (2010). Third, we redetect with high $\mathrm{S} / \mathrm{N}(\sim 30)$ the sharp extended feature (labeled as “3”) previously observed by Millan-Gabet \& Monnier (2002) with Keck at $J$ band (shown in white contours in Fig. 2, central panel). This feature extends down to 0.' 4 and appears spatially connected with the clump, and is likely to extend south beyond our field of view because of its high $\mathrm{S} / \mathrm{N}$ ( 28 at 1 1.'5 south). The relative polarized color (Fig. 2) shows that, in scattered light, feature " 3 " is remarkably redder when compared to the neutral $\left(\Delta m_{H-K_{\mathrm{s}}} \sim 0\right)$ polarized halo or with the bright clump $\left(\Delta m_{H-K_{\mathrm{s}}} \sim\right.$ $0.4)$. Figure 2 also suggests that feature " 3 " is more extended in the east-west direction in the $K_{\mathrm{s}}$ band than in the $H$ band, especially towards the south. In both bands the polarized flux along the feature decreases with projected distance to the star, but not uniformly. There is a local decrement in $P_{I}$ at $\sim-1{ }^{\prime \prime} 3$ south (see Fig. 2, central panel), also observed in intensity at the $J$ band in the Keck images. We find the same complex structures when processing the observations using different amounts of IP correction, and therefore conclude that they are not artifacts.

The polarization angle $P_{\theta}$ at $K_{\mathrm{s}}$ band is shown in Fig. 3, left panel. The vectors are plotted only in regions of the image with $S / N>10$ averaging over a 3 px width $\left(\sim 1.2\right.$ FWHM at $K_{\mathrm{s}}$ band $)$ squared box. Overall, the vectors follow an axisymmetric pattern around the ZCMa binary system and they show the same orientation along feature " 3 ". The white arrows indicate polarized artifacts created by the AO system. In these regions $P_{\theta}$ differs from the rest of the image, showing a nonaxisymmetrical orientation. An extension towards the east seems to depart from the polarized clump (see Fig. 2, central panel). This feature is more likely an artifact, since it is located very close to one AO artifact, and it does not appear in any of the other images. A zoom around the stars is shown in the central and right panels of Fig. 3. In these plots we only show $P_{\theta}$ where the $S / N>30$.

Interestingly, there is a polarized filament connecting the clump (feature "2") with the innermost regions of ZCMa. The bright pixels around the non-linear (set to 0) pixels are likely to have a contribution from the inherent noise observed in the inner $0 . ' 1$ around the stars (see the $P_{I}$ image of the comparison star at the bottom-right panel in Fig. 1). However, the two stars and their respective circumstellar disks (and cocoon around the primary) are known to be polarized (Fischer et al. 1998), and a large contribution from them and the polarized halo can explain the quasi-axisymmetric distribution of $P_{\theta}$, especially at the $K_{\mathrm{s}}$ band.

\section{Discussion and conclusions}

Our polarized observations show the complex and rich environment of ZCMa. The nature of feature "3" remains unclear. It is not spatially close to the two jets detected by Whelan et al. (2010), and to date there are no detections of jets in continuum (while there are several detections in emission lines). Particularly intriguing is the apparent change in direction it follows before connecting to the bright clump, and the morphological similarities with broad cylindrical cavities recently observed in CO at late stages of star formation (Lee \& Ho 2005; Arce et al. 2013) and at $10 \mu \mathrm{m}$ around massive embedded stars (Li et al. 2014). Although it is difficult to imagine how feature " 3 " can produce scattered light at $\leq 1400$ au (projected distance) from the stars, this feature could be explained by a large filament of dust with different scattering properties from its surrounding material. Alternatively, it could be created by light scattered off the walls of a cavity created by the interaction of a molecular outflow with the surrounding material. We find no change in the shape of that feature when comparing it with the Keck images taken 14 years ago (Fig. 2, central panel). Given the youth of $\mathrm{ZCMa}\left(\leq 3 \times 10^{5} \mathrm{yr}\right.$, van den Ancker et al. 2004) and the size of the polarized clump (feature " 2 "), a tantalizing explanation for this clump could be to relate it to an embedded, very young star. High-resolution images at mid-infrared wavelengths can help to disentangle the nature of this clump by providing evidence of a mid-IR excess (or lack thereof).

We detect orbital motion on the binary system of ZCMa. Assuming a circular orbit composed of a $1 M_{\odot}$ and a $5 M_{\odot}$ star and comparing it with previous measurements, our data suggest an almost face-on orbit with a measurable orbital motion of $\triangle \mathrm{PA}=0.7 \mathrm{yr}^{-1}$, in agreement with the values derived by Millan-Gabet \& Monnier (2002). The derived relative photometry is consistent with a new outburst from the system. 


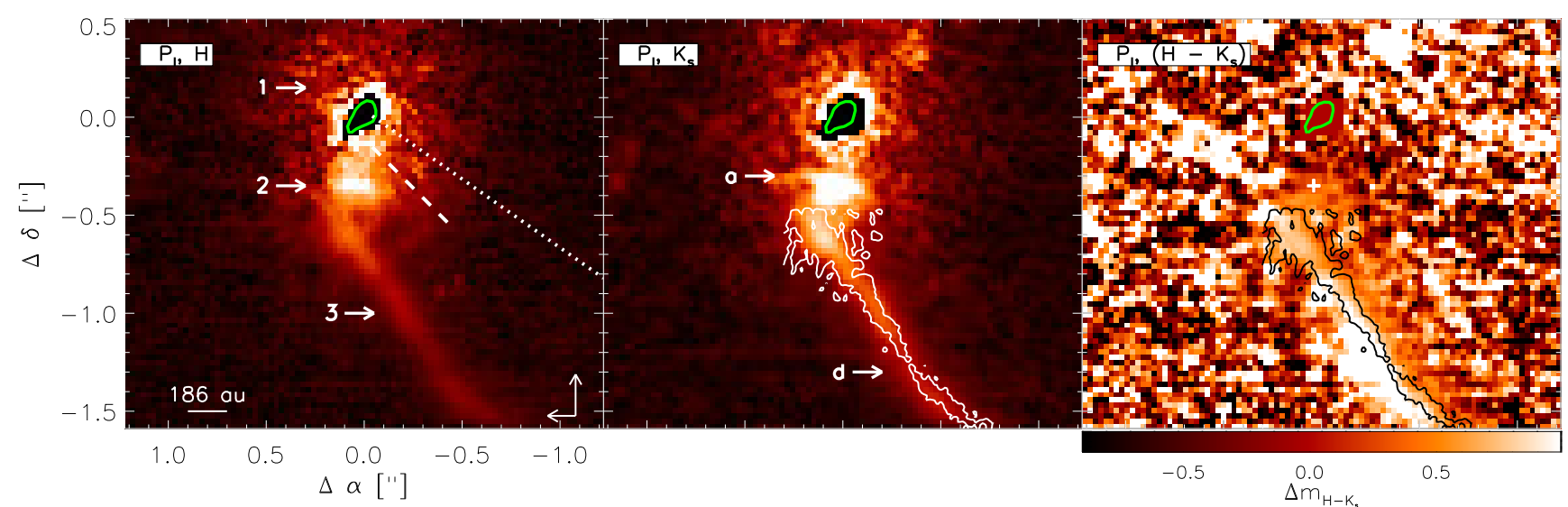

Fig. 2. Right and center: $P_{I}$ images at the $H$ and $K_{\mathrm{s}}$ bands. Green contours indicate the position of the two stars. The two images are plotted in the same scale, using square root scaling. The pixels falling in the non-linear regime are set to zero. Left: the dotted and dashed lines indicate the position of large and micro jets, respectively (Whelan et al. 2010). Arrows "1", "2", and " 3 " indicate the polarized halo, the bright clump, and the sharp extended feature, respectively. Center: Arrows "a" and "d" show an artifact and a local decrement in polarized flux. The white line contours the Keck $J$-band image presented in Millan-Gabet \& Monnier (2002). Right: relative polarized color computed as $[H]-\left[K_{\mathrm{s}}\right]$. The white cross indicates the brightest point of feature " 2 " at the $K_{\mathrm{s}}$ band.
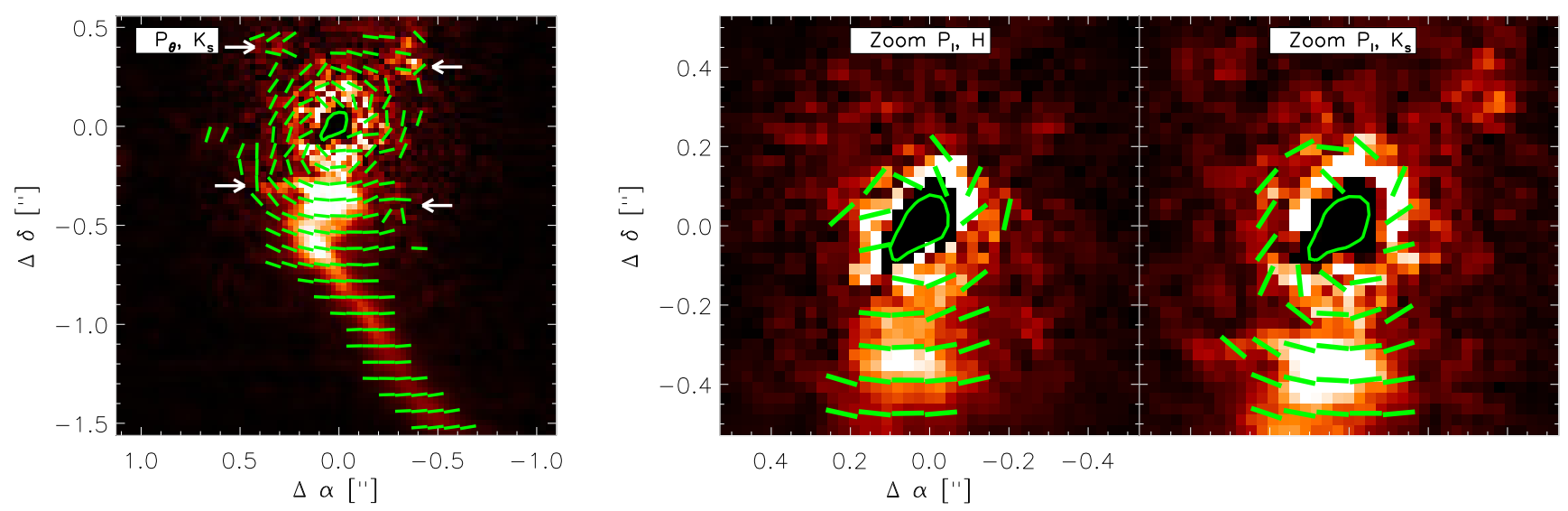

Fig. 3. Left: $P_{\theta}$ over polarized intensity at the $K_{\mathrm{s}}$ band. The length of the vectors is arbitrary. The image is plotted in linear scale, stretching the scale to highlight faint features. AO artifacts are indicated by the arrows.

The $1 \mathrm{~mm}$ and $3 \mathrm{~mm}$ continuum images obtained by Alonso-Albi et al. (2009) with IRAM-PdBI show a partially resolved structure elongated in the N-S direction that could be related to the observations here presented. In all cases, images at $\mathrm{mm}$ and sub-mm wavelengths with much higher spatial resolution are urgently needed to study the cold-dust counterpart of our observations to complete our picture of Z CMa and to understand the earliest evolutionary stages of the binary systems. Finally, our results show that NaCo's polarimetric imaging capabilities are at their best, allowing us to probe the innermost regions of $\mathrm{ZCMa}$

Acknowledgements. We are grateful to George Hau and to the ESO staff for their help during the observations, and to J. Monnier for sharing the Keck $J$-band images. This research was funded by the Millennium Science Initiative, Chilean Ministry of Economy, Nucleus RC130007. H.C. and C.C. acknowledge support from ALMA/CONICYT (grants 31100025 and 31130027). S.P. and S.C. acknowledge financial support provided by FONDECYT grants 3140601 and 1130949. C.C. and M.R.S. acknowledge support from CONICYTFONDECYT grant 3140592 and FONDECYT grant 1141269, respectively. L.C. was supported by ALMA-CONICYT and CONICYT-FONDECYT 31120009 and 1140109 .

\section{References}

Alonso-Albi, T., Fuente, A., Bachiller, R., et al. 2009, A\&A, 497, 117 Arce, H. G., Mardones, D., Corder, S. A., et al. 2013, ApJ, 774, 39 Avenhaus, H., Quanz, S. P., Schmid, H. M., et al. 2014, ApJ, 781, 87
Beckwith, S. V. W., \& Sargent, A. I. 1991, ApJ, 381, 250

Benisty, M., Malbet, F., Dougados, C., et al. 2010, A\&A, 517, L3

Canovas, H., Rodenhuis, M., Jeffers, S. V., Min, M., \& Keller, C. U. 2011, A\&A, 531, A102

Canovas, H., Min, M., Jeffers, S. V., Rodenhuis, M., \& Keller, C. U. 2012, A\&A, 543, A70

Canovas, H., Ménard, F., Hales, A., et al. 2013, A\&A, 556, A123

Clariá, J. J. 1974, A\&A, 37, 229

Fischer, O., Stecklum, B., \& Leinert, C. 1998, A\&A, 334, 969

Hinkley, S., Oppenheimer, B. R., Soummer, R., et al. 2009, ApJ, 701, 804

Hinkley, S., Hillenbrand, L., Oppenheimer, B. R., et al. 2013, ApJ, 763, L9

Kaltcheva, N. T., \& Hilditch, R. W. 2000, MNRAS, 312, 753

Koresko, C. D., Beckwith, S. V. W., Ghez, A. M., Matthews, K., \& Neugebauer, G. 1991, AJ, 102, 2073

Kraus, A. L., Ireland, M. J., Hillenbrand, L. A., \& Martinache, F. 2012, ApJ, 745,19

Lee, C.-F., \& Ho, P. T. P. 2005, ApJ, 624, 841

Lenzen, R., Hartung, M., Brandner, W., et al. 2003, Proc. SPIE, 4841, 944

Li, D., Mariñas, N., \& Telesco, C. M. 2014, ApJ, 796, 74

Millan-Gabet, R., \& Monnier, J. D. 2002, ApJ, 580, L167

Poetzel, R., Mundt, R., \& Ray, T. P. 1989, A\&A, 224, L13

Rousset, G., Lacombe, F., Puget, P., et al. 2003, Proc. SPIE, 4839, 140

Szeifert, T., Hubrig, S., Schöller, M., et al. 2010, A\&A, 509, L7

van den Ancker, M. E., Blondel, P. F. C., Tjin A Djie, H. R. E., et al. 2004, MNRAS, 349, 1516

Whelan, E. T., Dougados, C., Perrin, M. D., et al. 2010, ApJ, 720, L119

Whitney, B. A., \& Hartmann, L. 1993, ApJ, 402, 605

Whitney, B. A., Clayton, G. C., Schulte-Ladbeck, R. E., et al. 1993, ApJ, 417, 687

Witzel, G., Eckart, A., Buchholz, R. M., et al. 2011, A\&A, 525, A130 